DOI: https://doi.org/10.46296/yc.v5i8edespen.0088

\title{
ESTRATEGIAS DEL OCÉANO AZUL Y SU APLICABILIDAD EN EL CONTEXTO GERENCIAL ECUATORIANO
}

\section{STRATEGIES OF THE BLUE OCEAN AND ITS APPLICABILITY IN THE ECUADORIAN MANAGEMENT CONTEXT}

\author{
Padilla Greys Mairs ${ }^{1 *}$; Vegas-Meléndez Hilarión José ${ }^{2}$ \\ ${ }^{1}$ Licenciada en Administración Comercial, Universidad de Carabobo. Valencia - \\ Venezuela. \\ ${ }^{1}$ Docente e investigador de pre y posgrado de la Pontificia Universidad Católica del \\ Ecuador, sede Manabí. Portoviejo - Ecuador.
}

*Correo: gpadilla2578@pucesm.edu.ec

\begin{abstract}
Resumen
La investigación tiene como objetivo principal contextualizar los principios estratégicos del gerente ecuatoriano que dan sustento a los mercados alternativos denominados océano azul, permitiendo interpretar los descriptores gerenciales que emergen luego de analizar los escenarios favorables a las ideas empresariales; la investigación es de perspectiva cualitativa y de nivel descriptivo, con una intencionalidad heurística y de metodología inductiva; en las técnicas de recolección de información se encuentran la observación directa y la entrevista en profundidad, aplicándose a representantes del mundo empresarial, académicos e investigadores de reconocida trayectoria profesional en el mundo de la consultoría empresaria; la estrategia de análisis de la información parte desde la propuesta diseñada por la Teoría Fundamentada. Los resultados obtenidos muestran revisión de los conceptos sobre estrategias gerenciales, innovación y diferenciación en el marco del contexto empresarial ecuatoriano, así como sus modos de diseño frente a la posibilidad de crear nuevos mercados basados en la teoría del océano azul; como conclusión principal se puede develar una nueva concepción discursiva e interpretativa de la actuación gerencial y sus estrategias de abordaje a los océanos azules; además de la necesidad de aplicabilidad de descriptores gerenciales emergentes basados en análisis hermenéutico de la investigación.
\end{abstract}

Palabras claves: Estrategias, gerencia, océano azul, innovación, diferenciación.

\begin{abstract}
The main objective of the research is to contextualize the strategic principles of the Ecuadorian manager that give support to the alternative markets called blue ocean, allowing to interpret the managerial descriptors that emerge after analyzing the favorable scenarios for business ideas; the research is from a qualitative perspective and descriptive level, with a heuristic intention and inductive methodology; Information gathering techniques include direct observation and in-depth interviews, applied to representatives of the business world, academics and researchers with recognized professional trajectories in the world of business consulting; the information analysis strategy starts from the proposal designed by Grounded Theory. The results obtained show a revision of the concepts on managerial strategies, innovation and differentiation within the framework of the Ecuadorian business context, as well as their design modes against the possibility of creating new markets based on the theory of the blue ocean; As a main conclusion, a new discursive and interpretive conception of managerial action and its strategies for approaching the blue oceans can be revealed; in addition to the need for applicability of emerging managerial descriptors based on hermeneutical analysis of research.
\end{abstract}

Keywords: Strategies, management, blue ocean, innovation, differentiation.

Información del manuscrito:

Fecha de recepción: 16 de noviembre de 2020.

Fecha de aceptación: 08 de enero de 2021.

Fecha de publicación: 29 de enero de 2021. 


\section{Introducción}

Las estrategias juegan un rol importante para el éxito de cualquier actividad 0 plan que se desee desarrollar, en virtud de que las mismas permiten alcanzar los objetivos planificados. Chan y Mauborgne (2005) reflexionan sobre los tipos de mercados, logrando distinguirlos en océanos azules y rojos. Dentro de la propuesta del océano azul, las estrategias son referenciadas como innovadoras y diferenciadoras con relación a lo usual o cotidiano. Las posibilidades de una empresa para iniciar un nuevo océano azul, o de romper los esquemas actuales en un mar rojo, está supeditada a las estrategias gerenciales que visibilicen esta posibilidad. Esto último da origen al objetivo principal de esta investigación, orientando sus directrices en contextualizar los principios estratégicos del gerente ecuatoriano que dan sustento a los mercados alternativos denominados océano azul, tal como lo proponen Chan y Mauborgne (2005); así como visibilizar el pensamiento conceptual y estratégico sobre su aplicabilidad en el contexto ecuatoriano. La propuesta del océano azul en realidad -en primera instancia- no busca competir con el océano rojo, por el contrario, busca crear y desarrollar nuevos mercados que permitan brindar espacios para la diferenciación y la búsqueda constante de la innovación. Entre los elementos claves de las estrategias del océano azula es la identificación de los factores que no son visibles actualmente, tener como ventaja un espacio desconocido por los posibles competidores y, el tener las condiciones para crear una demanda. Esto se puede lograr con emprendimientos totalmente extraordinarios, únicos, irrepetibles, incomparables -o muy difíciles- de igualar por otros emprendedores; y otra es permanecer o incursionar en un océano rojo (mercado saturado) y de manera astuta (estrategias y tácticas) provocar un rompimiento de las reglas existentes que provoquen un cambio en los límites impuestos por los grandes del mercado.

Estas estrategias del océano azul han sido presentadas por sus proponentes como una forma de abandonar los mercados saturados, conocidos como "sangrientos y en constante guerra" (Chan y 
Mauborgne, 2005, p.10) entre empresas de un mismo ramo o servicio; este mercado es denominado océano rojo. Es decir, las empresas pueden crear océanos azules de forma inteligente, responsable y comprometida para maximizar sus oportunidades y minimizar los riesgos en sus operaciones; permitiendo establecer la posibilidad de crear nuevos mercados - con espacios seguros e innovadores- basados en la diferenciación y el bajo costo que representa.

La importancia del estudio de estas nuevas corrientes de abordaje hacia nuevos mercados basadas en la diferenciación, se puede observar claramente una ausencia del conocimiento sobre las actuales tendencias gerenciales que vislumbran el quehacer estratégico de quienes tienen la responsabilidad de pensar estratégicamente y el diseñar las acciones consecuentes que permitirán tener ventajas en mercados sin explorar; además de la necesidad de generar descriptores gerenciales que permitan ser guía para aquellos gerentes que buscan incursionar en nuevos mercados 0 en su defecto dar sustentabilidad a ideas de emprendimiento que generen nuevas propuestas para captación de consumidores leales y dejar de lado a quienes no se preocupan por la diferenciación sino por el precio; sin dudas, valorar una acción que esté vinculada al diseño de estrategias que den viabilidad a fuentes de atracción de los productos o servicios que se ofrecen.

El propósito de la investigación es el develar los descriptores estratégicos que bien permiten ir más allá de la demanda: Generar nuevas oportunidades de mercados alternativos; para ello, es imprescindible identificar las tácticas que bien pueden superar las franjas fronterizas de los actuales mercados, además de poseer las destrezas necesarias para romper esquemas tradicionales. Empresas de gran prestigio han asumido el rol de innovadores al incursionar en este océano azul, mostrando las posibilidades de crear nuevos espacios para el consumo, donde incluso el consumidor o usuario desconoce sobre las necesidades que el mercado está por crearle, y en consecuencia la empresa promotora de la nueva necesidad está dispuesta a satisfacer la demanda 
generada. De igual forma empresas tradicionales han virado su navegación en procura de nuevos y mejores momentos ante la lucha desproporcionada que se da en los denominados océanos rojos, en la que se observan competencias sin precedentes; en un océano azul, la competencia pierde su validez porque las reglas del juego todavía no existen. Frente a esta posibilidad investigativa se pretende abrir espacios para a un nuevo constructo teórico sobre las oportunidades reales de emprendimiento empresarial o en la redefinición de las actuales empresas en el territorio ecuatoriano, lo cual permita mostrar las potencialidades de los mercados existentes e incluso en la aparición de nuevos espacios de mercado. La investigación se permite mostrar una categorización conceptual basada en la saturación teórica, la triangulación discursiva de los informantes y de la interpretación por parte de los investigadores.

Ante esta reflexión de la temática, la investigación se plantea la siguiente interrogante: ¿Qué principios estratégicos del océano azul le permiten al gerente ecuatoriano desarrollar oportunidades de mercados alternativos? A fin de dar respuesta a la interrogante planteada la investigación se establece como objetivo principal el contextualizar los principios estratégicos del gerente ecuatoriano que dan sustento a los mercados alternativos denominados océano azul, procurando describir los principios estratégicos que dan sustento a los mercados de océano azul, así como el analizar el significado de mercados alternativos desde las posturas del océano azul en el contexto ecuatoriano; con ello, se procura una propuesta heurística de encontrar conceptos que bien permitan comprender el comportamiento de los gerentes ecuatorianos y su habilidad de establecer estrategias innovadoras que le permitan desarrollar oportunidades de mercados alternativos.

\section{Definiciones que sustentan un nuevo constructo}

Diversas definiciones pueden generar un nuevo constructo que bien permita avanzar teóricamente sobre las nuevas características que deben sobresalir de las ideas de emprendimiento que buscan crear un océano azul en base sus 
conceptos de diferenciación e innovación; sin embargo, se hace necesario la estructuración de estrategias orientadas en ambas direcciones -diferenciación e innovación- la cual viabilice su propuesta.

El término estrategia trae a relucir un sin número de situaciones que bien merecen ser contextualizadas según la necesidad de su aplicabilidad. Esta contextualización se refiere al ámbito en el cual se requiere hacer uso de la estrategia, en este caso en la gerencia. Ahora bien, las empresas conducidas por gerentes estrategas tienen una alta posibilidad de llegar a tener y sostener el éxito de sus actividades empresariales, lo cual por supuesto da ventajas frente a los mercados internos o externos. Estos mercados en ocasiones están "habitados" por empresas que buscan para sí mismos un alto grado de competitividad que les permita hacer frente a sus competidores, intentando minimizarse mutuamente casi de manera sangrienta. Para ello, los gerentes utilizan sus mayores conocimientos en materia de estrategia a fin de ganar espacios dentro el mercado, o al menos no perderlos. En este sentido, se define estrategia como la "determinación conjunta de objetivos de la empresa y de las líneas de acción para alcanzarlas" (Chandler, 1962, p.17); así como también tenemos que "las estrategias son los medios por los cuales se lograrán los objetivos" (David, 2013, p.62). Otra dimensión conceptual sobre la estrategia es cuando se describe como "el patrón o plan que integra las principales metas y políticas de una organización, y a la vez establece, la secuencia coherente de las acciones a realizar" (Mintzberg y Quinn, 2005, p. 42). Se puede considerar que una estrategia es exitosa cuando la gerencia encuentra el espacio (momento) adecuado para intentar imponer sus tácticas de manera controlada y en los tiempos requeridos.

Ahora bien, para imponer estas tácticas es necesario conocer el comportamiento del mercado además de poseer las destrezas necesarias para romper esquemas tradicionales, y más aún para intentar crear un mercado con características similares a la propuesta del océano azul.

Según W. Chan Kim y Renee Mauborgne (2018), los océanos 
azules son "... espacios de mercado no aprovechados y por la creación de demanda y oportunidades para un crecimiento altamente rentable", además de representar "las industrias que no existen actualmente. Es el espacio desconocido del mercado". Sin dudas, un aspecto relevante dentro de los océanos azules es y será la innovación. En la medida de que un producto o un servicio se muestre como innovador y diferenciado ante un potencial nuevo consumidor 0 cliente se podrá obtener un éxito casi de inmediato en posicionarlo en ese nuevo mercado. Es importante resaltar lo expresado por Gómez (2017) quien define la diferenciación en términos de producto como "el distinguir un producto o servicio del resto de sus competidores, buscando hacerlo más atractivo a los ojos de su mercado objetivo, para que lo prefieran" (p.99)

Un ejemplo con relación al párrafo anterior es lo expuesto por López, Carvallo1 y Jimbo (2018) en su artículo publicado "La Estrategia del Océano Azul y su relación con el Negocio Inclusivo del reciclaje en la ciudad de Cuenca, Ecuador", allí desarrollan la idea que el océano azul genera la oportunidad de inclusión social a la población con menores ingresos, iniciando la introducción del Negocio Inclusivo; aportando un novedoso esquema diferenciador. Es un aporte significativo dado que permite develar como se pueden desarrollar ideas innovadoras que benefician (oportunidad laboral) no solo a personas en situación vulnerable, sino también quienes padecen discapacidades; con ello se establece una oportunidad de inclusión en todas sus expresiones.

Al ser la innovación un condicionamiento importante para la creación de océanos azules es importante destacarla en el contexto teórico, para ello se consideran las siguientes posturas: Schumpeter (1934), la define "como un proceso de destrucción creativa, que permite que la economía y los agentes económicos evolucionen; asimismo, es la forma en que la empresa administra sus recursos a través del tiempo y desarrolla competencias que influyen en su competitividad". La UNESCO (1977) plantea que "la innovación involucra el empleo de los resultados de la investigación fundamental y aplicada en la 
introducción de nuevas aplicaciones o en la mejora de aplicaciones ya existentes". El Manual de Oslo (2005) establece que la innovación "es la introducción de un nuevo, o significativamente mejorado producto (bien o servicio), de un proceso, de un nuevo método de comercialización o de un nuevo método organizativo al mercado y la sociedad". Por su parte Drucker (1985) argumenta que "la innovación sistemática consiste en la búsqueda, organizada y con un objetivo, de cambios y en el análisis sistemático de las oportunidades que ellos pueden ofrecer para la innovación social o económica". Estos elementos teóricos permiten establecer una relación directa con los principios 1 y 3 de las estrategias del océano azul: Reconstruir las fronteras del mercado / Ir más allá de la demanda existente.

En el caso de la diferenciación es necesario la definición de estrategias basadas en ella; en ese aspecto de las estrategias se debe partir de un marco conceptual que bien defina los tipos de diferenciación que debe tener proyectar una empresa frente a sus competidores; para ello, es importante contar con el aporte de
Porter (2015) quien indica que "los dos tipos básicos, combinados con el ámbito de las actividades que las empresas intentan obtenerlos, dan origen a tres estrategias genéricas para lograr un desempeño superior promedio de la industria: 1) liderazgo en costos, 2) diferenciación en los costos y 3) enfoque en la diferenciación"; el mismo Porter (2015) sostiene que la empresa "intenta distinguirse dentro de su sector industrial en aspectos bastante apreciados por los compradores. Escoge uno o más atributos que juzgue importantes y adopta un posicionamiento especial para atener esas necesidades. Ve premiada su singularidad con su mayor precio" (p.41).

Por su parte, Gómez (2017) resalta que la diferenciación "es distinguir un producto o servicio del resto de sus competidores, buscando hacerlo más atractivo a los ojos de su mercado objetivo, para que lo prefieran" (pág. 99), la clara idea de Gómez (2017), de que se debe evitar competir por precio es con la firme intención de "apartar el producto o servicio de los demás, poniéndolo en otra categoría. No es comparable. EI valor que representa para el cliente 
supera su relación costo - beneficio por encima de la competencia" (pág. 100). Esto brinda una connotación muy peculiar al producto o servicio que se presta, obteniéndose como resultado una segmentación como bien lo acotan Chirinos Cuadros \& Rosado Samaniego (2016) al expresar que "en los mercados actuales existen segmentos con altas exigencias de servicio que se constituyen en una oportunidad", siendo esta oportunidad convertida en un factor diferencial que al final tampoco es muy duradera dada la evolución continua que tienen los mercados que se basan justamente en la innovación para la diferenciación, allí Gómez (2017) deja muy en claro que "los diferenciales son transitorios. La estrategia necesita evolución continuamente, porque los clientes y los mercados están cambiando más rápido que nunca”. (pág. 192).

\section{Aspectos filosóficos. Aportes para la discusión}

Sin duda, las estrategias aplicadas para el desarrollo del mercado basado en las características del océano azul jugaran un papel importante en el nuevo constructo. Entre los elementos claves es la identificación de los factores que no son visibles actualmente, tener como ventaja un espacio desconocido por los posibles competidores $y$, por último, tener las condiciones para crear demanda por parte de los usuarios 0 posibles clientes, evitándose así la disputa de estos. Ello se puede lograr con emprendimientos totalmente novedosos, únicos, irrepetibles, imposibles -o muy difíciles- de igualar por otros emprendedores; y otra es permanecer o incursionar en un océano rojo (mercado saturado) y de manera astuta (estrategias y tácticas) provocar un rompimiento de las reglas existentes que provoquen un cambio en los límites impuestos por los grandes del mercado.

La investigación se desarrolló bajo la premisa de establecer una triangulación de posturas entre actores importantes en el contexto empresarial ecuatoriano, cuya propuesta heurística fue la de encontrar elementos o variables que permitan comprender el comportamiento de los gerentes ecuatorianos y su habilidad de establecer estrategias innovadoras para que sus empresas se consoliden, así como el buscar 
nuevas alternativas que les permitan pasar de un estado de inacción a un estado de mayor desarrollo de oportunidades frente a los vientos cada vez más cambiantes que hacen vida en los mercados tanto nacionales como internacionales. A través de un proceso de categorización, la investigación devela una identidad epistémica gracias a la reflexión epistemológica única de los investigadores, la cual se soporta por una cohesión entre los aspectos filosóficos propios de la investigación, dado a entender desde lo siguiente:

Desde los aspectos filosóficos en lo siguiente: Ontológico, se procura entender la esencia del gerente estratégico; en lo axiológico, desvelar los valores predominantes en la interacción del gerente y el mercado; lo epistemológico, revelar la estructura conceptual de cual se parte los términos gerentes, estrategias e innovación; en lo metodológico conducirse por el sendero de lo inductivo-interpretativo permitiendo configurar un nuevo constructo teórico-conceptual sobre gerencia estratégica y su comportamiento frente a los mercados saturados o sin explorar, mostrando nuevas oportunidades. Por último, la utilización de la técnica de análisis de la información empírica recogida en las entrevistas en profundidad propuesta por la Teoría Fundamentada facilitó la construcción de los datos para la posterior categorización de los descriptores emergentes en la investigación.

En lo socioeconómico se puede considerar como una propuesta para la focalización de esfuerzos económicos y financieros en ideas empresariales realmente sostenibles, y por demás sustentables. Gerentes y emprendedores podrán visibilizar con más detalles los elementos condicionantes que se deben tener en cuenta para el momento de hacer una inversión, la cual debe estar directamente vinculada con su propósito estratégico o filosofía empresarial, donde esta a su vez identifique la razón de ser frente a los mercados.

En lo académico, la investigación busca reflexionar sobre el tema y la necesaria incorporación de su debate en los espacios de enseñanza y aprendizajes; además contribuye al consolidar la línea de 
investigación: Administración y Aseguramiento de la Calidad del Programa Maestría en Administración de Empresas de la Pontificia Universidad Católica del Ecuador como institución propulsora de estudios de posgrado, en la que se busca fortalecer la práctica investigativa a través de la publicación de trabajos científicos.

En definitiva, el presente trabajo de investigación aporta una metódica para la discusión académica, dando alcance de los objetivos propuestos desde la perspectiva propia de la investigación; a su vez proporciona una categoría de descriptores estratégicos a ser utilizados por quienes deseen incursionar en el océano azul desde su empresa constituida 0 idea de emprendimiento; y no menos importante una estructura discursiva reflexiva por parte de los exponentes gerenciales sobre las realidades del mercado y los retos a los cuales se enfrentan actualmente, así como sus propuestas frente al futuro.

4. Océano azul en el Ecuador. Una metódica de abordaje

La investigación centra su perspectiva interpretativa del discurso gerencial en el contexto ecuatoriano, para ello se genera un rastreo investigativo que permite referenciar sobre bases sólidas los argumentos expuestos por quienes de alguna manera son los hacedores de cambios a través de las decisiones que se toman en el ámbito empresarial, como lo son los gerentes.

Se plantea una arquitectura metodológica de enfoque cualitativo y de nivel descriptivo, estableciendo en primera instancia la observación directa por parte del investigador, estableciendo una intencionalidad heurística e inductiva, argumentada en postulados teóricos y referentes documentales, la revisión de los hechos y su posterior contraste. Esto permite viabilidad a una conducta interpretativa del discurso gerencial por parte de los investigadores; valorando los argumentos expuestos -a través de la entrevista- dado por los actores protagonistas generadores del cambio empresarial, así como de conocer la postura de expertos académicos e investigadores estudiosos de la temática analizada, en este caso las estrategias del océano azul. 
La entrevista en profundidad se establece como instrumento principal de recolección de información; por otro lado, se considera como estrategia de análisis de la información suministrada por los actores 0 informantes la técnica propuesta por Strauss \& Corbin (2002) desde la Teoría Fundamentada en la que se obtiene en el proceso una categorización que facilita el proceso de generación de una teoría sustantiva, peos la posterior teorización formal de la investigación; claro está, que esta parte de la investigación solo procura alcanzar la categorización de los descriptores gerenciales que facilitan el proceso de transición hacia un mercado de océano azul.

En cuanto a la entrevista en profundidad, se puede considerar que esta busca:

Obtener las concepciones personales de los entrevistados sobre la situación objeto de la investigación en medio de un esfuerzo de inmersión $y$ reinversión con intimidad $y$ familiaridad para sinceramente reconstruir a través de la discursividad el punto de vista (perspectiva) del informante (o actor relevante) en el cual se ubica la investigación y su contexto (Vegas, 2016, pág. 79)

Cabe destacar que los actores que se consideran válidos para el suministro de información relevante son gerentes de alto prestigio en el mundo empresarial ecuatoriano, entre los que podemos mencionar al Presidente de la Asociación Nacional de Empresarios (ANDE), además de académicos como la Dra. Amelia Escalona (PhD) y el Dr. Víctor Hugo Pinto (PhD), ambos catedráticos de la Universidad de Carabobo (Venezuela) investigadores expertos en la temática y consultores gerenciales de importantes empresas y emprendedores, su participación en la investigación permite conocer una visión foránea sobre los océanos azules y su comportamiento en mercados globales. A fin de tener una ordenación conceptual dentro de los expuesto por los informantes es necesario establecer un recorrido para la comprensión, interpretación y aplicación de lo manifestado por estos mismos informantes en términos hermenéuticos, tal como lo propone Gadamer (1998). 
Se aporta una metódica para el alcance de los objetivos propuestos, además de brindar una categoría de acciones estratégicas cuyo norte es el mercado de océano azul, en el cual se encuentre la empresa o idea de emprendimiento. La investigación además hace un aporte al consolidar la línea de investigación calidad y productividad, así como al área de conocimiento administración; de esta forma se busca configurar un constructo de la conducta estratégica y el discurso gerencial, estableciendo nuevos descriptores gerenciales basados en la innovación y en la diferenciación.

En entrevista realizada al Presidente de la Asociación Nacional de Empresarios del Ecuador (ANDE), Sr. René Ortiz (2020) destaca "la necesidad que tiene la empresa ecuatoriana de abrirse aún más al mercado global, donde sus productos puedan medirse en iguales condiciones pero con una marcada calidad en los mismos", lo cual es un indicativo la necesidad de generación de conocimiento sobre los mercados a partir del mismo contexto empresarial, y en el caso de la gerencia "esta debe estar focalizada en la disciplina y la responsabilidad de la acción profesional, mostrando sentido de pertenencia, competencias probadas y por supuesto alto conocimiento del mercado" según el mismo Ortiz (2020).

Para Ortiz (2020), un gerente estratega ecuatoriano debe de "ser expertos en mercados internacionales, comportamiento global de los consumidores, administradores de nuevos espacios de consumo; allí estará el éxito de la empresa que hoy día lucha por sostenerse"; por ello es importante la necesidad de establecer mecanismos que viabilicen la transición a nuevos mercados aún no explorados, o como bien se expresa en nuevos espacios de consumo que no son visibles a simple vista.

Un componente importante en la conversación, fue el denominado discurso gerencial desde la perspectiva del océano azul; para el Ortiz (2020) "el discurso gerencial, sin embargo, es vacío, no muestra avances de inserción en temas de innovación y búsqueda de nuevos proceso de aprendizaje, lo cual nos coloca en situación de desventaja frente a otros mercados"; esta 
preocupación es válida ante la incorporación acelerada de nuevas tecnologías que buscan ser incorporadas por muchas empresas, cuyo fin último es la ventaja competitiva, basada muchas de ellas en la diferenciación estratégica; además en relación al papel de la academia y los espacios para el debate reflexivo sobre los temas de actualidad, en especial los relacionados con la discurso diferenciador que marca la pauta en los nuevos mercados, allí Ortiz (2020) manifiesta que "la universidad juega un papel importante en la elaboración del discurso empresarial, y más aún cuando egresan profesionales con alta formación, pero con debilidades discursivas que no nos permiten reflexionar sobre las bases del nuevo contexto empresarial en el Ecuador", ello sin dudas marca las necesidades que se deben abordar desde lo académico, a fin de facilitar el la construcción de nuevas posturas discursivas por parte de los gerentes ecuatorianos.

Frente a la visión global del gerente ante las nuevas rupturas que se dan en los mercados, para la investigadora Escalona (2020) un gerente "debe ser más que estratega, debe ser visto como un agente de cambio para la empresa. Sus ideas siempre están orientadas a la innovación y el futuro del mercado, la ruptura siempre va a estar latente. El gerente se anticipa." En este caso Pinto (2020) resalta que "las rupturas en los mercados son necesarias porque permiten al consumidor encontrar alternativas que nacen producto de la innovación, y los océanos azules son un buen ejemplo de ello; es una buena oportunidad para modelar tus propios consumidores". Otro aspecto interesante en la conversación (entrevista) fue la incorporación del término "valorarse" pero no bajo la figura conocida dentro de la denominada cadena de valor de un producto o servicio, sino en cómo se auto identifica la empresa frente al mercado; acá Escalona es muy precisa al comentar que el valorarse "es reconocerse a sí mismo en la empresa como generador de un producto o servicio que le cambia la vida de manera sustancial al consumidor, y este último reconoce la diferencia frente a otros en el mercado"; Pinto (2020) alerta un poco sobre el tema de la diferenciación al considerar "que el 
peor enemigo de la diferenciación, es la <guerra de precios> que siempre busca imponerse, su mejor aliada es la situación económica de la cual pueda padecer el consumidor, sacrificando calidad por precio. Aunque tenga clara la diferencia".

Un elemento abordado y que representa un aspecto importante a considerar por toda empresa, es el de focalizar adecuadamente al consumidor; Escalona (2020) sostiene que "las empresas en su mayoría quieren siempre vender desmedidamente sin considerar las perspectivas, emociones 0 necesidades reales del consumidor, convirtiéndolas en empresas frías y calculadoras; en muchas ocasiones el consumidor quiere le reconozcan su fidelidad"; por su parte Pinto (2020) agrega que es necesario "pensar en componentes diferenciales que procuren el reconocimiento a la lealtad por parte del consumidor, evitándoles dudas sobre alternativas diferentes. Un buen diferenciador nace desde los consumidores, ellos son los que dan valor a la diferenciación". Otro aspecto abordado fue el de las estrategias. Estas son sin dudas la clave para cualquier transición al océano azul. Escalona (2020) sentencia que las estrategias "no pueden ser ejecutadas en base a suposiciones, estas deben de tener un alto sentido de entendimiento y conocimiento pleno del mercado que se quiere crear, con ello se obtendrá la ventaja difícil de perder"; en la misma idea de las estrategias Pinto (2020) manifiesta que una estrategia de diferenciación bien diseñada "permitirá ir hacia donde está la oportunidad, evitando la acostumbrado "necesidad" de enfrentar al competidor, en un espacio del mercado que ya este domina".

No cabe la menor duda que los elementos expuestos en las diferentes entrevistas generan un nuevo espacio para el constructor de descriptores relacionados a las estrategias del océano azul y que bien pueden ser tomados en consideración en el contexto ecuatoriano. El surgimiento de estos descriptores resultantes se da luego del proceso de categorización desarrollado en el análisis hermenéutico por parte de los investigadores. 
La siguiente tabla 1 muestra, de manera muy resumida, las categorías y descriptores resultantes luego del análisis realizado por los investigadores, en ella se observan las categorías según el perfil del entrevistado, y los descriptores que resultan luego del cruce entre las categorías que se asemejan entre sí.

Tabla 1. Categorías y Descriptores Emergentes de la Entrevista en Profundidad.

\begin{tabular}{|c|c|c|c|}
\hline Entrevistado & Códigos en vivo & Categorías & Descriptores \\
\hline $\begin{array}{l}\text { AC: } \quad \text { Rene } \\
\text { Ortiz (2020) }\end{array}$ & $\begin{array}{l}\text { - Gerente con visión de } \\
\text { mercados globales. } \\
\text { - Competencias. } \\
\text { probadas ante los } \\
\text { mercados } \\
\text { - Ausencia de innovación } \\
\text { y nuevos aprendizajes. } \\
\text { - Discurso ajustado al } \\
\text { nuevo contexto } \\
\text { empresarial. }\end{array}$ & $\begin{array}{l}\text { - Mercados } \\
\text { globales. } \\
\text { - Innovación. } \\
\text { - Aprendizaje. } \\
\text { - Discurso. } \\
\text { - Sustentabilidad } \\
\text { del mercado } \\
\text { local. }\end{array}$ & $\begin{array}{l}\text { - Mercado diferenciado y } \\
\text { sustentable. } \\
\text { - Modelo autónomo de } \\
\text { gestión empresarial. }\end{array}$ \\
\hline $\begin{array}{l}\text { EAl: Amelia } \\
\text { Escalona } \\
(2020)\end{array}$ & $\begin{array}{l}\text { - Gerente visionario, } \\
\text { futurista. } \\
\text { - Factor de cambios de } \\
\text { comportamiento. } \\
\text { - Fidelidad valorada. } \\
\text { - Diferenciador emerge, } \\
\text { no se impone. }\end{array}$ & $\begin{array}{l}\text { - } \text { Crear futuro } \\
\text { presente. } \\
\text { - Co-diseño de } \\
\text { una identidad } \\
\text { propia. } \\
\text { - Vinculación } \\
\text { estratégica. }\end{array}$ & $\begin{array}{l}\text { - Negocios basados en } \\
\text { el concepto de la } \\
\text { diferenciación. } \\
\text { - Estrategias de mercado } \\
\text { basadas en la } \\
\text { innovación y la } \\
\text { diferenciación holística. }\end{array}$ \\
\hline $\begin{array}{l}\text { EAl. } \\
\text { Hugo } \\
(2020)\end{array}$ & $\begin{array}{l}\text { - Cliente modelado, no } \\
\text { cazado. } \\
\text { - Entorno económico } \\
\text { influyente. } \\
\text { - Consumidores. } \\
\text { participes del concepto } \\
\text { de negocio. } \\
\text { - Acción basada en } \\
\text { oportunidades y no en } \\
\text { necesidades. }\end{array}$ & $\begin{array}{l}\text { - Valor } \\
\text { compartido del } \\
\text { significado } \\
\text { calidad. } \\
\text { - Cerrar la brecha } \\
\text { con el cliente } \\
\text { - Reconocimiento } \\
\text { del entorno. }\end{array}$ & $\begin{array}{l}\text { - Simbiosis negocio- } \\
\text { cliente. } \\
\text { - Consumidores } \\
\text { "educados". }\end{array}$ \\
\hline
\end{tabular}

Fuente: Padilla \& Vegas (2020) a partir de entrevistas en profundidad.

Notas: AC: Actor de cambio; EAl: Experto Académico Investigador.

Tal como se señala en la tabla anterior los descriptores emergentes del análisis establecen una ruta a seguir como parte del comportamiento que bien se puede asumir por quienes tienen la responsabilidad de diseñar estrategias basadas en la innovación y la diferenciación, cuya intensión está en una transición hacia los océano azules, y que sin dudas buscan sacar ventajas frente a 
situaciones que otros posibles competidores aún no imaginan o simplemente no se encuentran aptos para competir, sino para ser seguidores.

Los descriptores estratégicos que emergen en la investigación son los siguientes:

Negocios basados en el concepto de la diferenciación. Permite una idealización que habla más allá del producto o servicio que se presta, dejando claro la diferencia entre oportunidad y necesidad.

Modelo autónomo de gestión empresarial. El conocer cada espacio del mercado permite crear un modelo autónomo basado en su cultura empresarial en la que juega un papel importante la filosofía del cambio en su transición al océano azul.

\section{Mercado diferenciado y} sustentable. Una empresa que llega a crear un océano azul rápidamente establece las condiciones en las que la innovación y la diferenciación se van a ajustar gradualmente; dando ventajas ilimitadas frente a quienes llegan posteriormente.
Estrategias de mercado basadas en la innovación y la diferenciación holística. Para una adecuada formulación de estrategias es necesaria una asociación de igual forma estratégica con los clientes que "han nacido" en la transición y que pueden contribuir para su diseño y aprobación, fortaleciendo la fidelidad por parte de cliente ante esta nueva forma de asociación.

Simbiosis negocio-cliente. En la transición al océano azul, entre el cliente y la idea de negocio se puede dar una simbiosis en la que ambos se necesitan. Crecen juntos y protegen el mercado ante ataques de seguidores competidores- que buscan imponer una guerra de precios.

Consumidores “educados". Es una condición de suma importancia en las que las empresas de océano azul deben invertir mucho tiempo y dinero. Mostrando las virtudes diferenciadoras del producto 0 servicio desde las perspectivas de la calidad, uso e impacto de orden personal o profesional. De esta forma se obtiene un consumidor que representa el concepto de la 
empresa en cada accionar individual o profesional.

Estos descriptores son producto de la reflexión discursiva de quienes tienen la posibilidad de aportar en beneficio de la gerencia estratégica, específicamente en la viabilidad de una transición hacia el océano azul.

\section{Desde la orilla del océano azul}

Por su parte, y de manera reflexiva, la investigación establece sus conclusiones tomando en consideración los elementos que orbitan alrededor de la propuesta del objetivo principal en la que se establece la necesidad de contextualizar los principios estratégicos del gerente ecuatoriano que dan sustento a los mercados alternativos denominados océano azul; para ello se recogen los argumentos que se develan desde los hallazgos resultantes -mostrados en los descriptores expuestos- y que generan desde la presente investigación las siguientes consideraciones.

1.- Es necesario un discurso ajustado a las nuevas realidades del emprendimiento empresarial, en la que se destaque como la sustentabilidad del negocio generará la incorporación de nuevos productos y servicios en mercados no explorados; es necesario un constructo que garantice que la idea de negocio no fracase al no entender las condiciones para transitar hacia un mercado de océano azul. Es necesario construir un discurso estandarte sobre la responsabilidad social empresarial y de la corresponsabilidad ciudadana, con ello se gana identidad e identificación.

2.- Los mercados de océano azul requiere de descriptores estratégicos diferenciados desde la perspectiva de cada empresa. Cada idea de negocio parte de un diferenciador base o genérico; sin embrago, debe aprender a construir su propio espacio diferenciador, su conveniente segmento del mercado, el adecuado uso de los datos para contextualizar las estrategias; sin esa habilidad no podrá mostrarse ante un consumidor en potencia, dado que no es capaz de mostrarle un producto o servicio que aún este mismo consumidor no sabe que necesita o le será útil. 
3.- Sin dudas, es importante una estructura organizativa que contribuya en la diferenciación y el costo, facilitando el proceso de transición hacia los océanos azules a través de la competencia creativa y la innovación, ambos como ejes fundamentales del gerente estratega en su aspiración de abordar nuevas alternativas de emprendimiento en mercados inexistentes.

La investigación cierra un espacio...otro está por abrirse.

\section{Bibliografía}

Chan Kim, W. y Mauborgne R. (2018). La transición al océano azul. Como desarrollar un nuevo mercado done la competencia no tiene ninguna importancia. Grupo Editorial ACTIVA

Chan Kim, W. y Mauborgne R. (2005). La estrategia del océano azul. Como desarrollar un nuevo mercado done la competencia no tiene ninguna importancia. Grupo Editorial Norma. https://www.researchgate.net/ publication/318682060

Chandler, A. (1962). Strategy and Structure: Chapters in the History of Industrial Enterprise. Group Doubleday. https://papers.ssrn.com/sol3/ papers.cfm?abstract_id $=1496$ 188

David, F. (2013). Conceptos de administración estratégica. Editorial Pearson, novena edición.

https://maliaoceano.files.word press.com/2017/03/libro-freddavid-9a-edicion-conestrategica-fred-david.pdf

Drucker, P. (1985). Innovation \& Entrepreneurship: Practice and Principles. Editorial Butterworth Heinemann.

Escalona A. (2020). Estrategias del océano azul y su aplicabilidad en el contexto gerencial ecuatoriano. Entrevista en profundidad.

Gadamer, H. G. (1998). Fundamentos de la Hermenéutica. Siglo XX Editores.

Gómez, D. (2017). Bueno, bonito y carito. Editorial Penguin Random House Grupo Editorial.

López, G., Carvallo, L. S. y Jimbo, J. S. (2018). La Estrategia del Océano Azul y su relación con el Negocio Inclusivo del reciclaje en la ciudad de Cuenca. Revista MASKANA. Ecuador. https://doi.org/10.1 8537/mskn.09.02.03

Manual de Oslo. (2006). Guía para la recogida e interpretación de datos sobre la innovación. 
Organización de Cooperación y Desarrollo Económicos. OECD y Eurostat. Tercera Edición.

Mintzberg, E., y Quinn, B. (2005). El proceso estratégico. Conceptos, contextos y casos. Prentice Hall Hispanoamericana, S.A. https://isabelportoperez.files. wordpress.com/2012/08/proc eso_estrategico_mintzberg.p df.

Ortiz, R. (2020). Estrategias del océano azul y su aplicabilidad en el contexto gerencial ecuatoriano. Entrevista en profundidad.

Pinto, V.H. (2020). Estrategias del océano azul y su aplicabilidad en el contexto gerencial ecuatoriano. Entrevista en profundidad.

Porter, M. E. (2015). Ventaja Competitiva: Creación y sostenimiento de un desempeño superior. México: Grupo Editorial Patria, S.A De C.V. https://books.google.com.ec/ books?id=wV4JDAAAQBAJ\& printsec=frontcover\&dq=vent aja+competitiva+de+michael +porter\&hl=es\&sa=X\&ved $=2 a$ hUKEwiTzIGznK_sAhWHjVk KHfvXAOcQ6AEwAHoECAU

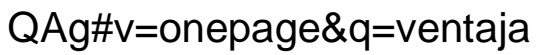
\%20competitiva\%20de\%20mi chael\%20porter\&f=false
Schumpeter, J. (1978). Teoría del desenvolvimiento económico. Fondo de Cultura Económica.

Strauss, A. y Corbin, J. (2016). Bases de la investigación cualitativa. Técnicas y procedimientos para desarrollar la teoría fundamentada. (2a. ed.) CONTUS-Editorial, Universidad de Antioquia.

UNESCO (1977). Innovación y problemas de la educación: teoría y realidad en los países en desarrollo. https://unesdoc.unesco.org/ar k:/48223/pf0000136018

Vegas, H. (2016). La teoría fundamentada como herramienta metodológica para el estudio de la gestión pública local. Revista Venezolana de Gerencia., 21(75).

https://produccioncientificaluz .org/index.php/rvg/article/view /21891/21636 